\title{
Outbreak of varicella in preschool children despite one-dose vaccination
}

\author{
Zafer Kurugöl ${ }^{1}$, Şule Gökçe ${ }^{2}$ \\ Division of ${ }^{1}$ Pediatric Infectious Diseases and ${ }^{2}$ General Pediatric Unit, Department of Pediatrics, Ege University Faculty \\ of Medicine, Izmir, Turkey.E-mail: zafer.kurugol@ege.edu.tr \\ Received: 25th April 2017, Revised: 29th June 2017, 31st July 2017, Accepted: 17th September 2017
}

SUMMARY: Kurugöl Z, Gökçe Ş. Outbreak of varicella in preschool children despite one-dose vaccination. Turk J Pediatr 2018; 60: 56-62.

In Turkey, a single-dose varicella vaccine was introduced into the National Immunization Program in 2013. Before this implementation, varicella vaccine had been available in the private sector since 2000 . However, varicella outbreaks continued to occur in preschools and elementary schools. We investigated a varicella outbreak to estimate the effectiveness of 1-dose varicella vaccine and to evaluate potential risk factors for breakthrough disease. This study was carried out during a varicella outbreak in 3 preschools in İzmir, Turkey, in April 2016. Using questionnaires, data including children's medical and vaccination histories were collected from their parents. Attack rates in vaccinated and unvaccinated children were calculated and the analyses of vaccine effectiveness and of risk factors for breakthrough disease were conducted.

A total of 124 children were enrolled in the study. Of the 124 children, 77 $(62 \%)$ had received 1-dose varicella vaccine before the outbreak. Varicella developed in 34 of 124 children during the outbreak, and 18 of them (53\%) had breakthrough varicella. The attack rate was $23.4 \%$ among vaccinated children and $34 \%$ among unvaccinated children. The effectiveness of singledose varicella vaccine was $33.6 \%$ against varicella disease of any severity and $82.5 \%$ against moderate or severe varicella. Children vaccinated 5 or more years before the outbreak had 3.5 times the risk of disease than those who had been vaccinated more recently (OR 3.5 [95\% CI, 1.08-11.5]); $\mathrm{p}=0.046$ ). Age at vaccination ( $<15$ months vs. $\geq 15$ months) and the brands of varicella vaccine were not associated with the increased risk of breakthrough varicella.

Our study suggests that one-dose of varicella vaccine is not sufficient to prevent school outbreaks. A 2-dose varicella vaccination program may help to prevent varicella outbreaks and achieve effective control of the disease.

Key words: varicella vaccine, breakthrough varicella, vaccine effectiveness, outbreak, preschool children.

A varicella vaccine, based on the attenuated live varicella zoster virus (VZV) Oka strain, was first developed by Dr. Takahashi in Japan in 1974. ${ }^{1}$ Today, varicella vaccines, all contain the live attenuated VZV Oka strain, are available in many countries and have been introduced into the National Immunization Program in some developed countries. The United States is the first country to implement the varicella vaccine into the national immunization program. After the implementation of the single -dose varicella vaccination to children aged 12-15 months in 1996, varicella incidense, varicella-related hospitalization and mortality have dramatically decreased $^{2}$. However, despite high vaccination coverage, outbreaks of varicella continue to occur in day-care centers and schools among single-dose-vaccinated children. ${ }^{3-5}$ For this reason, in order to control the outbreaks and interrupt endemic disease transmission, the United States changed from a routine 1-dose varicella vaccination program to a universal 2-dose program (first dose at 12-18 months and booster at $4-6$ years) in 2006.6 Similarly, today a 2-dose schedule has also been recommended in many developed countries such as Germany, 
Japan and Canada. ${ }^{7}$ After the implementation of the routine 2 -dose program in the countries, further reductions have been seen in both varicella disease burden and the number of outbreaks. $^{8-9}$

In Turkey, two brands of monovalent varicella vaccine (Okavax Sanofi-Pasteur and VarilrixGlaxoSmithKline) have been available in the private sector since 2000. In 2013, a single-dose varicella vaccine was included in the National Immunization Program for children 12 months of age and vaccination coverage of $95 \%$ or more has been reached in children 12-36 months of age. Although Turkish children have been routinely immunized with the single-dose varicella vaccine for more than three years, and in spite of high vaccination coverage, varicella cases and varicella outbreaks are still ongoing, especially in preschools and elementary schools. However, the data of how many of these varicella cases are vaccinated children and the data of their vaccination status is insufficient in our country. We investigated an outbreak of varicella among children attending preschools to estimate the effectiveness of 1-dose varicella vaccine, and to evaluate potential risk factors for breakthrough disease.

\section{Material and Methods \\ Study population and design}

This study was carried out during a varicella outbreak in 3 preschools in İmir, Turkey, in April 2016. Previously healthy children with no immune deficiency or history of a chronic disease were enrolled in the study. Children who had a previous history of varicella disease were excluded from the study. Their parents were interviewed and they filled out a questionnaire about their children's medical history and demographic characteristics. Varicella vaccination status of the children was determined by examining their immunization records. A written informed consent was obtained from parents before enrolling patients.

Children with suspected varicella were examined by a pediatrician in the first 3 days of the illness. A case of varicella was clinically

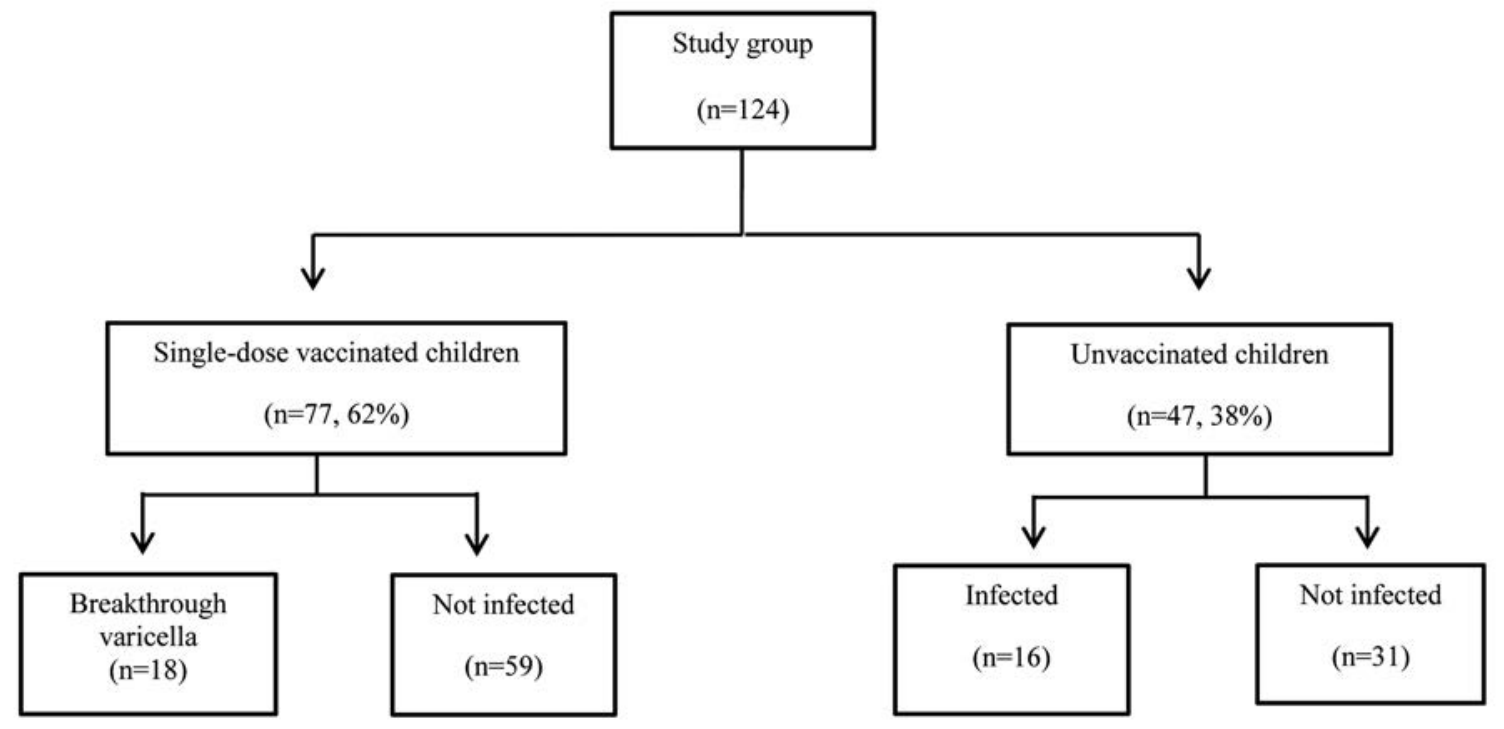

* The attack rates of varicella among single-dose vaccinated and unvaccinated children were $23.4 \%$ and $34 \%$, respectively.

** Single-dose varicella vaccine effectiveness is $33.6 \%$ against any disease and against $82.5 \%$ moderate and severe disease.

Fig. 1. Vaccination and varicella infection status of the children in the study group. 
Table I. Clinical Characteristics of Illness Among Single-dose Vaccinated Children with Varicella and Unvaccinated Children with Varicella.

\begin{tabular}{clll}
\hline & $\begin{array}{l}\text { Breakthrough varicella } \\
\text { cases }(\mathrm{n}=18)\end{array}$ & $\begin{array}{l}\text { Unvaccinated cases } \\
(\mathrm{n}=16)\end{array}$ & $\mathrm{p}$ \\
\hline Severity of illness, n (\%) & & & \\
\multicolumn{1}{c}{ Mild* } & $16(89)$ & $9(56.3)$ & 0.031 \\
$\quad$ Moderate/severe** & $2(11)$ & $7(43.7)$ & \\
Presence of fever, n (\%) & $2(11.1)$ & $9(56.3)$ & 0.021 \\
Duration of disease (mean $\pm \mathrm{SD})$ & $7.6 \pm 1.9$ & $7.7 \pm 1.5$ & 0.574 \\
\hline
\end{tabular}

* Mild disease: fever than 50 lesions without complication

** Moderate disease: 51-500 lesions without complication; severe disease: more than 500 lesions or presence of any complications

diagnosed through an acute, generalized, maculopapulovesicular rash without other apparent cause in a child. A varicella outbreak was defined as $\geq 5$ cases of varicella occurred in a preschool within one week $(\mathrm{Fu})$. Breakthrough varicella was defined as varicella infection in a child who had been vaccinated more than 42 days before the onset of rash. Varicella disease was classified as mild ( $<50$ lesions), moderate (51-500 lesions without any complications) and severe ( $>500$ lesions or presence of any serious complications or hospitalization).

All procedures performed in studies involving human participants were in accordance with the ethical standards of the institutional and/or national research committee and with the 1964 Helsinki declaration and its later amendments or comparable ethical standards. The local ethics committee approved this study.

\section{Statistical analysis}

Statistical analysis was performed using SPSS for Windows (version 21.0). Characteristics of vaccinated vs. unvaccinated children were compared using t-test and Mann Whitney U test for continuous variables and Chi-square test for categorical variables. To evaluate the potential risk factors for breakthrough disease, the logistic regression (univariate) model was used and odds ratio was calculated. A p value of $<0.05$ was considered statistically significant.

The attack rates among unvaccinated children (ARU) and vaccinated children (ARV) were calculated. Then vaccine effectiveness (VE) was calculated using the equation: [(ARU-ARV)/ ARU] x $100^{10}$. The effectiveness of the varicella vaccine was determined for all varicella disease as well as for moderate/severe disease.

\section{Results}

This study was carried out in three preschools where the outbreak of varicella was seen. A total of 179 children, who were previously healthy attending these preschools, were enrolled in the study. Fifty-three of them had a history of varicella, so the 53 children were excluded from the study, leaving 126 children. Of the 126 children, 79 had received varicella vaccine before the outbreak. Two of the vaccinated children had received 2 dose of varicella vaccine; they were excluded from the study. Thus, 124 children were included in the study.

Among the 124 children, 77 (62\%) had been vaccinated with a single dose of varicella vaccine and $47(38 \%)$ were unvaccinated (Fig. 1). A total of 34 cases of varicella were observed during the outbreak: 18 cases (53\%) occurred in single-dose vaccinated children (breakthrough varicella cases) and 16 cases $(47 \%)$ in unvaccinated children. The attack rates of varicella were $23.4 \%$ among singledose vaccinated children and $34 \%$ among unvaccinated children. During the outbreak, the effectiveness of single-dose varicella vaccine was $33.6 \%$ against varicella of any severity and $82.5 \%$ against moderate or severe varicella.

Overall, 25 cases of varicella were mild, and 9 cases were moderate or severe. No cases developed severe complications nor required hospitalization. Only one unvaccinated child developed keratitis. As compared with unvaccinated cases, breakthrough varicella cases had milder disease and were less likely to have fever (Table I). Eighty-nine percent 
of single-dose vaccinated children had mild disease; whereas the disease was mild in $56.3 \%$ of the unvaccinated group $(\mathrm{p}=0.031)$. Fever $\left(>38.3{ }^{\circ} \mathrm{C}\right)$ was observed in $11.1 \%$ of breakthrough varicella cases and $56.3 \%$ of the unvaccinated cases $(p=0.021)$. The duration of varicella infection was similar between the two groups $(7.6 \pm 1.9$ vs. $7.7 \pm 1.5$ days, $\mathrm{p}=0.574)$.

The median time since vaccination before the outbreak was 51 months; 16 children had been vaccinated 5 or more years previously; while the time lapsed after vaccination was less than 5 years in 61 children.

Time since vaccination was significantly associated with the increased risk of breakthrough varicella (Table II). Children vaccinated 5 or more years before the outbreak had 3.5 times the risk of breakthrough disease than those vaccinated within 5 years (OR 3.5; 95\% CI: 1.08-11.5); $\mathrm{p}=0.046)$. The breakthrough varicella was observed in $18 \%$ of children who had been vaccinated at $<5$ years, and in $44 \%$ of those vaccinated at $\geq 5$ years. Among single-dose vaccinated 77 children, 67 were vaccinated before 15 months and 10 were vaccinated after 15 months and over. The age of varicella vaccination ( $<15$ months vs. $\geq 15$ months) were not associated with the increased of breakthrough disease in this outbreak. There were also no association between breakthrough varicella and the brands of varicella vaccine, administering solely varicella vaccine or the simultaneous administration with measlesmumps-rubella (MMR) vaccine.

\section{Discussion}

Studies carried out so far have shown that the effectiveness of a single-dose of varicella vaccine is mean $80 \%$ (ranged from 20 to $100 \%$ ) against varicella disease of any severity and mean $95 \%$ (ranged from 78 to $100 \%$ ) against moderate and severe disease. ${ }^{11,12}$ However, several studies have reported the single-dose varicella vaccine effectiveness as low as $20-72 \%$ during varicella outbreaks. ${ }^{3,13-18}$ In the present study, it was found that single-dose varicella vaccination provided poor protection against varicella disease of any severity during a varicella outbreak in the three preschools. The effectiveness of the single-dose varicella vaccine was found to be quite low; as $33.6 \%$ against varicella disease of any severity and $82.5 \%$ against moderate and severe varicella. The vaccinated children in our study group had been immunized with varicella vaccine procured from pharmacies or the private sector. For this reason, cold-chain problems such as storage, distribution, transport and application of vaccines might be associated with the low vaccine effectiveness. Another factor which may have negatively affected the

Table II. Risk Factors for Breakthrough Varicella Among Single-dose Vaccinated Children.

\begin{tabular}{|c|c|c|c|c|}
\hline & $\begin{array}{l}\text { Breakthrough } \\
\text { varicella }(\mathrm{n}=18)\end{array}$ & $\begin{array}{l}\text { Not infected } \\
(\mathrm{n}=59)\end{array}$ & OR $(95 \% \mathrm{CI}) *$ & $\mathrm{p}$ \\
\hline \multicolumn{5}{|l|}{ Age at vaccination, $\mathrm{n}(\%)$} \\
\hline$<15$ months & $16(24)$ & $51(76)$ & $1.25(0.24-6.5)$ & 0.763 \\
\hline$\geq 15$ months & $2(20)$ & $8(80)$ & & \\
\hline \multicolumn{5}{|l|}{ Time since vaccination, $\mathrm{n}(\%)$} \\
\hline$<5$ years & $11(18)$ & $50(82)$ & $3.5(1.08-11.5)$ & 0.046 \\
\hline$\geq 5$ years & $7(44)$ & $9(56)$ & & \\
\hline \multicolumn{5}{|l|}{$\begin{array}{l}\text { Time between varicella and MMR } \\
\text { vaccination*, n (\%) }\end{array}$} \\
\hline Simultaneous administration & $5(19)$ & $22(81)$ & & 0.818 \\
\hline Separate administration & $13(26)$ & $37(74)$ & $1.1(0.3-4.2)$ & \\
\hline \multicolumn{5}{|l|}{ Brand of varicella vaccines, $\mathrm{n}(\%)$} \\
\hline Varilrix & $6(26)$ & $17(74)$ & $1.7(0.3-9.3)$ & 0.542 \\
\hline Okavax & $3(38)$ & $5(62)$ & & \\
\hline Not known & $9(20)$ & $37(80)$ & & \\
\hline
\end{tabular}

* OR: Odds ratio, CI: Confidence interval

** MMR: Measles, mumps, and rubella 
vaccine effectiveness could be the widespread circulation of the virus during the outbreak in the crowded classes of preschools.

In the present study, time since vaccination was identified as a significant risk factor for breakthrough varicella. Children vaccinated 5 or more years before the start of the outbreak had a 3.5-fold higher risk for breakthrough disease than those who had been vaccinated less than 5 years before. Similarly, several studies have reported there was a significantly higher breakthrough disease risk ratios for children vaccinated over 5 years ago than for children vaccinated more recently. 3,13,14,17,19,20-22 However, some studies have found no association between time elapsed after vaccination and the risk of breakthrough varicella. ${ }^{16,23,24} \mathrm{~A}$ recent review published by Bonanni et al. ${ }^{25}$ reviewing primary and secondary failure after varicella vaccination showed that most cases of breakthrough varicella could be accounted for by primary vaccine failure. A study from Spain reviewing 10 years of breakthrough varicella in children vaccinated with one dose also showed that half of the breakthrough varicella cases happened within 2 years of vaccination. ${ }^{26}$ It has also been reported that the time interval between the administration of first and second doses does not affect the booster response; comparable antibody responses were obtained regardless of whether the second vaccine dose was given 6-12 weeks or 3-6 years after the first dose. ${ }^{9}$

Several studies reported that younger age at vaccination ( $\leq 15$ months) may be a risk factor for breakthrough varicella $4,21,27,28$. However, the evidence is also not consistent. Some studies found no association between age at vaccination and breakthrough varicella. ${ }^{13,16,20,22,23}$ In our study too, we determined that younger age at vaccination (less than 15 months) is not a risk factor for breakthrough varicella. We also found that the risk of breakthrough varicella is not associated with the vaccine brand administered. A study from Germany reported that the risk of breakthrough varicella was higher with some brands of varicella vaccine compared with others $^{16}$. However, in many other studies, no significant differences in breakthrough varicella rates were found between subjects who had received different vaccine brands, which was in agreement with our observations. It is not surprising that the effectiveness of varicella vaccines produced by different companies is similar because all varicella vaccines in the world, except the vaccine licensed in South Korea, are based on the Oka strain of VZV.

Breakthrough varicella infection has been described as clinically milder, with fewer lesions, complications, and systemic symptoms but recent studies report that breakthrough varicella disease can result in serious complications similar to those occurring in unvaccinated individuals. ${ }^{29-32}$ Although most cases of breakthrough varicella are mild, in our study, $11 \%$ of the cases had a moderate to severe disease. A prospective-hospital based varicella surveillance study (VARICOMP study) from Turkey reported that pediatric breakthrough varicella requiring hospitalization was not rare, $9 \%$ of the breakthrough varicella cases had severe varicella disease, and neurological complications such as encephalitis and meningitis were the most common reasons for hospitalization ${ }^{33}$. Moreover, children with breakthrough varicella can transmit the virus, although transmission is less frequent than from unvaccinated children with varicella. In school settings, risk of transmission increases further. ${ }^{13,23}$ For this reason, breakthrough varicella is an important health problem not only because it may cause complications and hospitalizations, but also for its potential for transmission.

In conclusion, one-dose of varicella vaccine does not provide enough protection to prevent outbreaks completely. Although it has been more than three years since implementation of the universal 1-dose varicella vaccination program in Turkey, and despite high vaccination coverage, varicella outbreaks continue to occur in preschools and elementary schools. In this outbreak, more than half of varicella cases were single-dose vaccinated children. From this point of view, to prevent varicella outbreaks and to further reduce the number of varicella cases, changing from a routine 1-dose varicella vaccination program to a routine 2-dose vaccination program seems to be logical in Turkey, as it is currently recommended by many countries. $6,7,9$ 


\section{Acknowledgements}

The authors would like to thank the study staff at the Statistical Analyses Department, and the infants and their families for participating in the study.

\section{REFERENCES}

1. Takahashi M. Effectiveness of live varicella vaccine. Expert Opin Biol Ther 2004; 4: 199-216.

2. Vázquez M, LaRussa PS, Gershon AA, Steinberg SP, Freudigman K, Shapiro ED. The effectiveness of the varicella vaccine in clinical practice. $\mathrm{N}$ Engl J Med 2001; 344: 955-960.

3. Tugwell BD, Lee LE, Gillette H, Lorber EM, Hedberg K, Cieslak PR. Chickenpox outbreak in a highly vaccinated school population. Pediatrics 2004; 113: 455-459.

4. Galil K, Fair E, Mountcastle N, Britz P, Seward J.Younger age at vaccination may increase risk of varicella vaccine failure. J Infect Dis 2002; 186: 102-105.

5. Lopez AS, Guris D, Zimmerman L, et al. One dose of varicella vaccine does not prevent school outbreaks: is it time for a second dose? Pediatrics 2006; 117: e1070-e1077.

6. Marin M, Güris D, Chaves SS, Schmid S, Seward JF; Advisory Committee on Immunization Practices, Centers for Disease Control and Prevention (CDC). Prevention of varicella: recommendations of the Advisory Committee on Immunization Practices (ACIP). MMWR Recomm Rep 2007; 56(RR-4): 1-40.

7. Rentier B, Gershon AA; European Working Group on Varicella. Consensu: varicella vaccination of healthy children-a challenge for Europe. Pediatr Infect Dis J 2004; 23: 379-389.

8. Kattan JA, Sosa LE, Bohnwagner HD, Hadler JL. . Impact of 2-dose vaccination on varicella epidemiology: Connecticut--2005-2008. J Infect Dis 2011; 203: 509512.

9. European Centre for Disease Prevention and Control (ECDC) GuIddance Varicella vaccination in the European Union. www.ecdc.europa.eu

10. Orenstein WA, Bernier RH, Hinman AR. Assessing vaccine efficacy in the field. Epidemiol Rev 1988; 10: 221-241.

11. Marin M, Marti M, Kambhampati A, Jeram SM, Seward JF. Global Varicella Vaccine Effectiveness: A Meta-analysis. Pediatrics 2016; 137: e20153741.

12. WHO. Strategic Advisory Group of Experts on Immunization (SAGE) Working Group on Varicella and Herpes Zoster Vaccines. SAGE on Immunization, 2 April 2014, Geneva.

13. Galil K, Lee B, Strine T, et al. Outbreak of varicella at a day care center despite vaccination. N Engl J Med 2002; 347: 1909-1915.

14. Lee BR, Feaver SL, Miller CA, Hedberg CW, Ehresmann KR. An elementary school outbreak of varicella attributed to vaccine failure: policy implications. J Infect Dis 2004; 190: 477-483.
15. Miron D, Lavi I, Kitov R, Hendler A. Vaccine effectiveness and severity of varicella among previously vaccinated children during outbreaks in day-care centers with low vaccination coverage. Pediatr Infect Dis J 2005; 24: 233-236.

16. Spackova M, Wiese-Posselt M, Dehnert M, MatysiakKlose D, Heininger U, Siedler A. Comparative varicella vaccine effectiveness during outbreaks in day-care centres. Vaccine 2010; 28: 686-691.

17. Arnedo-Pena A, Puig-Barberà J, Aznar-Orenga MA, et al. Varicella vaccine effectiveness during an outbreak in a partially vaccinated population in Spain. Pediatr Infect Dis J 2006; 25: 774-778.

18. Bayer O, Heininger U, Heiligensetzer C, von Kries $\mathrm{R}$. Metaanalysis of vaccine effectiveness in varicella outbreaks. Vaccine 2007; 17: 6655-6660.

19. Chaves SS, Zhang J, Civen R, et al. Varicella disease among vaccinated persons: clinical and epidemiological characteristics, 1997-2005. J Infect Dis 2008; 197(Suppl 2): S127-S131.

20. Lu L, Suo L, Li J, et al. A varicella outbreak in a school with high one-dose vaccination coverage, Beijing, China. Vaccine 2012; 20: 5094-5098.

21. Haddad MB, Hill MB, Pavia AT, et al. Vaccine effectiveness during a Varicella outbreak among schoolchildren: Utah, 2002-2003. Pediatrics 2005; 115: 1488-1493.

22. Kurugol Z, Halicioglu O, Koc F, Koturoglu G, Aksit $S$. Varicella rates among unvaccinated and one-dose vaccinated healthy children in Izmir, Turkey. Int J Infect Dis 2011; 15: e475-e480.

23. Fu J, Wang J, Jiang C, Shi R, Ma T. Outbreak of varicella in a highly vaccinated preschool population. Int J Infect Dis 2015; 37: 14-18.

24. Kuter B, Matthews H, Shinefield H; Study Group for Varivax. Ten year follow-up of healthy children who received one or two injections of varicella vaccine. Study Group for Varivax. Pediatr Infect Dis J 2004; 23: $132-137$.

25. Bonanni P, Gershon A, Gershon M, et al. Primary versus secondary failure after varicella vaccination: Implications for interval between 2 doses. Pediatr Infect Dis J 2013; 32: e305-e313.

26. Redondo Granado MJ, Vizcaino Lopez I, Garcia Saseta P, Torres Hinojal C, Nieto Sánchez R. Early presentation of breakthrough varicella in vaccinated children. An Pediatr (Barc) 2013; 78: 330-334.

27. Dworkin MS, Jennings CE, Roth-Thomas J, Lang JE, Stukenberg C, Lumpkin JR. An Outbreak of Varicella among children attending preschool and elementary school in Illinois. Clin Infect Dis 2002; 35: 102-104.

28. Verstraeten T, Jumaan AO, Mullooly JP; Vaccine Safety Datalink Research Group. A retrospective cohort study of the association of varicella vaccine failure with asthma, steroid use, age at vaccination, and measles-mumps-rubella vaccination. Pediatrics 2003; 112: 98-103. 
29. Bernstein HH, Rothstein EP, Watson BM, et al. Clinical survey of natural varicella compared with breakthrough varicella after immunizationwith live attenuated Oka/ Merck varicella vaccine. Pediatrics 1993; 92: 833-837.

30. Aslan A, Kurugol Z, Gokben S. Acute transverse myelitis complicating breakthrough varicella infection. Pediatr Infect Dis J 2014; 33: 1196-1198.

31. Schwab J, Ryan M. Varicella zoster virus meningitis in a previously immunized child. Pediatrics 2004; 114: e273-e274.
32. Kelley J, Tristram D, Yamada M, Grose C. Failure of a Single Varicella Vaccination to Protect Children With Cancer From Life-Threatening Breakthrough Varicella. Pediatr Infect Dis J 2015; 34: 1027-1029.

33. Dinleyici EC, Kurugol Z, Kara A, et al; VARICOMP Study Group. Children with breakthrough varicella infection requiring hospitalization in Turkey (VARICOMP Study 2008-2013). Vaccine 2015; 33: 3983-3987. 